\title{
DNA repair deficiency in neuropathogenesis: when all roads lead to mitochondria
}

\author{
Luis Bermúdez-Guzmán ${ }^{1}$ and Alejandro Leal ${ }^{1,2^{*}}$ (D)
}

\begin{abstract}
Mutations in DNA repair enzymes can cause two neurological clinical manifestations: a developmental impairment and a degenerative disease. Polynucleotide kinase $3^{\prime}$-phosphatase (PNKP) is an enzyme that is actively involved in DNA repair in both single and double strand break repair systems. Mutations in this protein or others in the same pathway are responsible for a complex group of diseases with a broad clinical spectrum. Besides, mitochondrial dysfunction also has been consolidated as a hallmark of brain degeneration. Here we provide evidence that supports a shared role between mitochondrial dysfunction and DNA repair defects in the pathogenesis of the nervous system. As models, we analyze PNKP-related disorders, focusing on Charcot-Marie-Tooth disease and ataxia. A better understanding of the molecular dynamics of this relationship could provide improved diagnosis and treatment for neurological diseases.
\end{abstract}

Keywords: DNA repair, Mitochondrial dysfunction, Neurodegeneration, Ataxia, PNKP

\section{Background}

The present review aims to expand knowledge in the pathological process that involves defects in DNA repair and its interaction with mitochondrial dysfunction in neurodegeneration. Recently, evidence has grown on the involvement of mitochondrial dysfunction in neurodegenerative diseases of the central nervous system, such as Alzheimer's and Parkinson's and peripheral nervous system as well. Our team has worked mainly on the molecular causes of peripheral neuropathies that are classified as Charcot-Marie-Tooth disease (CMT). We identified a group of patients with mutations in the PNKP gene, an essential nuclear and mitochondrial DNA repair enzyme. $\mathrm{Mu}-$ tations in this gene have been associated with a pathological spectrum, varying from a neurodevelopmental impairment to a neurodegenerative disease. In this review, we analyze the existing literature that supports a pathological interaction between DNA repair and mitochondria, that causes an exclusive neurological effect, as in the case of PNKP-associated diseases.

\footnotetext{
* Correspondence: alejandro.leal@ucr.ac.cr

${ }^{1}$ Section of Genetics and Biotechnology, School of Biology, Universidad de Costa Rica, San José 11501, Costa Rica

${ }^{2}$ Neuroscience Research Center, Universidad de Costa Rica, San José, Costa Rica
}

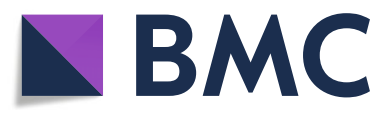

(c) The Author(s). 2019 Open Access This article is distributed under the terms of the Creative Commons Attribution 4.0 International License (http://creativecommons.org/licenses/by/4.0/), which permits unrestricted use, distribution, and reproduction in any medium, provided you give appropriate credit to the original author(s) and the source, provide a link to the Creative Commons license, and indicate if changes were made. The Creative Commons Public Domain Dedication waiver (http://creativecommons.org/publicdomain/zero/1.0/) applies to the data made available in this article, unless otherwise stated.

\section{Main text}

Nuclear and mitochondrial DNA are susceptible to damage due to errors of intrinsic DNA metabolism, and its exposure to radiation, reactive oxygen species and other environmental factors. Therefore, the DNA damage response is critical for cell survival and health [1].

The polynucleotide kinase 3 '-phosphatase (PNKP) is the main enzyme responsible for restoring the 5 '-phosphate and 3'-hydroxyl ends in DNA strand breaks, necessary for ligation during repair, especially in single strand breaks (SSBs) (Fig. 1) [4-6]. PNKP is recruited to repair errors in SSBs through interactions between its $\mathrm{N}$-terminal FHA domain and the X-Ray Repair Cross Complementing one (XRCC1) protein, necessary for the recruitment of PNKP and other proteins [7]. In the case of double-strand breaks (DSBs), PNKP participates, in the non-homologous end joining pathway (NHEJ), which takes place when the FHA domain interacts with the X-Ray Repair Cross Complementing 4 protein (XRCC4) [8]. However, it was also demonstrated that DSBs end-joining reaction could be dependent on PARP1, PNKP (hPNK) and the XRCC1/LIG III complex, known as alternative NHEJ [9]. 


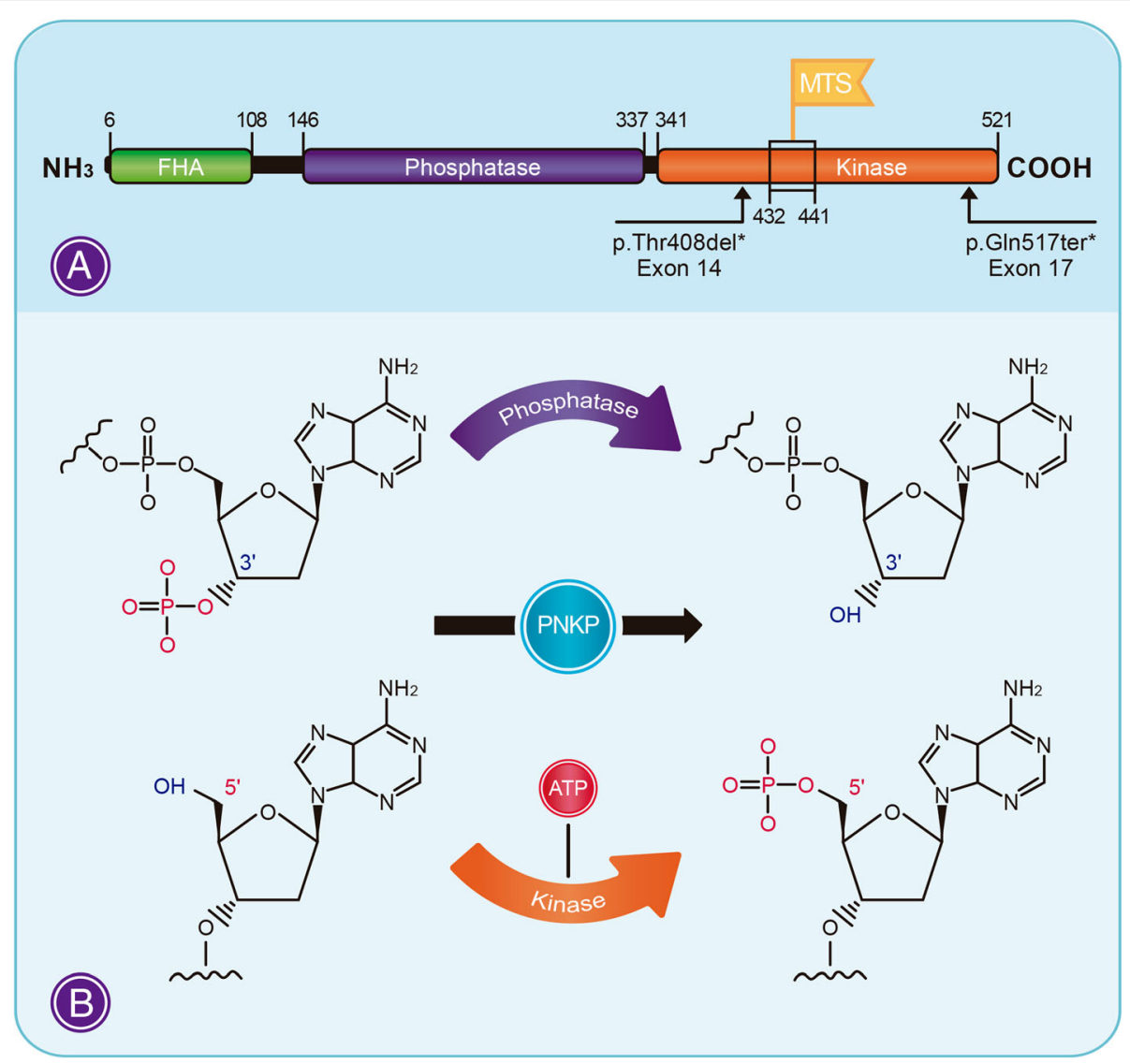

Fig. 1 a Schematic representation of the functional domains of PNKP. We reported five unrelated patients with CMT2 disease, who were compound heterozygotes for the p.Thr408del (exon 14) and p.GIn517ter * (exon 17) variants [2]. Homozygous individuals for the p.Thr408del variant present Ataxia-ocular motor apraxia 4 (AOA4) [3]. b Enzymatic role of PNKP. The enzyme catalyzes the 3'-phosphate termini dephosphorylation and the phosphorylation of $5^{\prime}$-OH terminal by using ATP as the phosphate donor. Mitochondrial-targeting signal (MTS) is a necessary region for PNKP translocation to mitochondria. FHA: Fork-head-associated domain

\section{SSB repair function in the nervous system: the model of} PNKP in health and disease

Due to its high rate of oxygen consumption, the nervous system is susceptible to DNA damage, particularly in comparison with other tissues that contain non-replicating cell types. The brain metabolizes $\sim 20 \%$ of consumed oxygen but has a low capacity to neutralize reactive oxygen species (ROS). Therefore, neurons are particularly susceptible to oxidative stress [10]. SSBs occur when one strand of the DNA losses a single nucleotide. If not repaired, it will represent a severe threat to genetic stability and cell survival. SSBs are considered the most common damage to DNA (1000 breaks/cell/day) in the cell [11], mainly derived from reactive oxygen species [12] and commonly repaired by the PNKP + XRCC1 complex. Since neurons have low levels of antioxidant enzymes and high transcriptional activity, these cells depend on the single-strand break repair pathway (SSBR) [13].

SSBR proceeds in four stages: break detection, processing of DNA termini, DNA gap filling and ligation
(Fig. 2). In the mature nervous system, genome stability related mechanisms differ from those operating during neurogenesis because of the lack of cell division and the absence of replicative DNA damage and homologous recombination repair [14]. Proliferating neural cells are proficient for DNA damage-induced apoptosis, [15] a resource to avoid excessive damage and control cell growth. In contrast, in post-mitotic cells, including neurons and cardiomyocytes, death by apoptosis is not that common and requires additional steps [16].

To complete the scenario, double-strand breaks (DSBs) can occur spontaneously in the brain, because of the high neuronal activity [17]. Nevertheless, in non-cycling cells, the homologous recombination system (HR) that repairs double-stranded breaks is unavailable [14]. Therefore, in post-mitotic neurons, NHEJ is the sole available pathway to repair this problem [18]. In the absence of DNA ligase IV, a key NHEJ component, neurons progressively accumulate endogenous DSBs [19]. Thus, in the mature nervous system, strand breaks and 


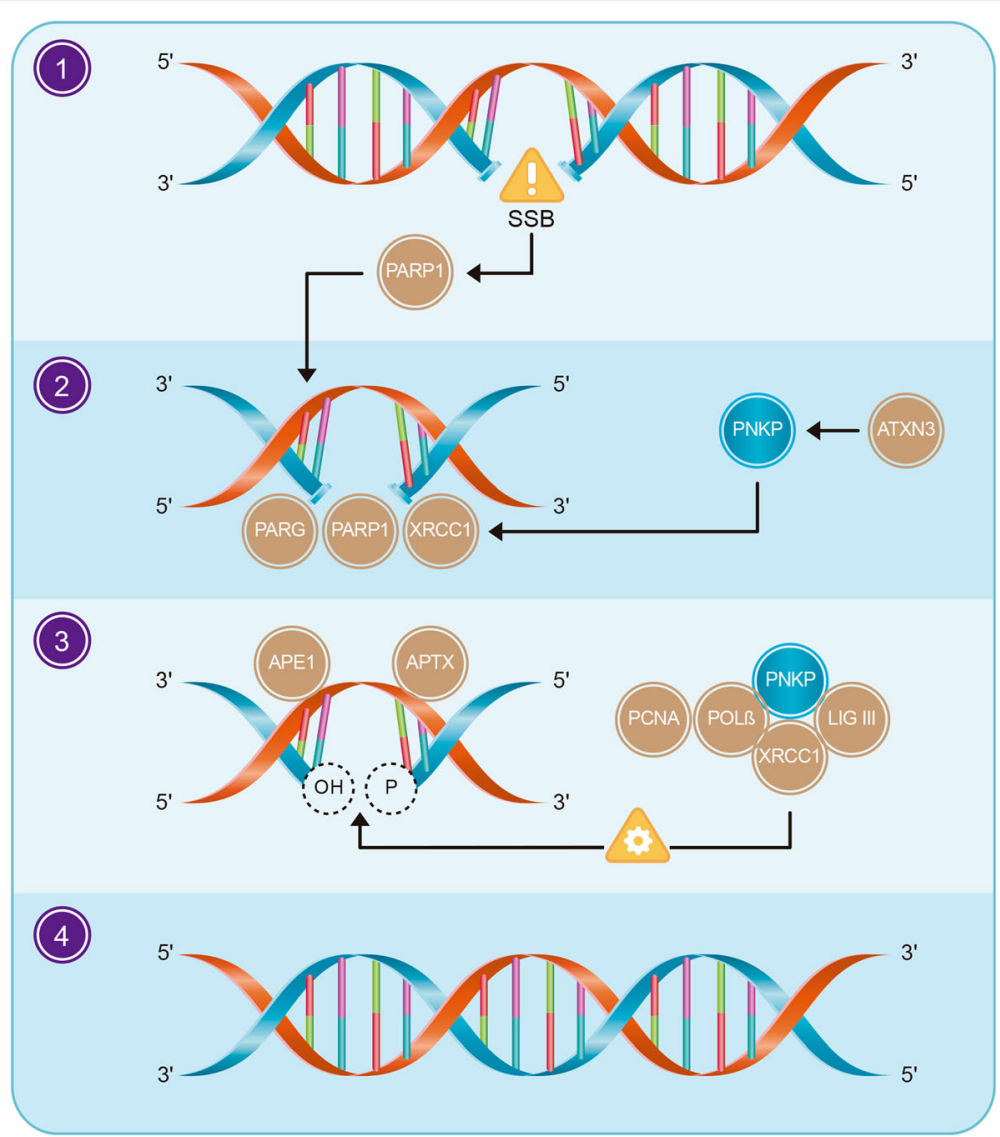

Fig. 2 Basic stages of Single-Strand Break Repair: 1. Detection of the break, 2. DNA end processing, 3. DNA gap filling and DNA ligation, 4. Repaired strand. Direct breaks are detected by PARP1, which promotes the accumulation of different repair factors. Damaged termini are repaired by APE1, DNA polymerase (Pol $\beta$ ), PNKP and APTX. Ligase (LIG III) is needed to repair short-patch sites through a phosphodiester bond

DNA modifications from oxidative damage can disrupt transcription and lead to cell death [20].

Despite the broad spectrum of diseases associated with mutations in DNA repair enzymes (such as immunodeficiency and higher cancer risk), defects in the SSBR pathway present a tendency to affect the nervous system, in some cases exclusively [21-23]. As PNKP has a vital role in both nuclear and mitochondrial DNA integrity, mutations in this enzyme or associated proteins, have several implications in the pathogenesis of neurological diseases. Until now, two different clinical manifestations have been associated with PNKP mutations: a neurodevelopmental impairment (Microcephaly and Seizures, MCSZ) and neurodegenerative disorders (i.e., Ataxia-ocular motor apraxia 4, AOA4) [24]. To illustrate the impact of DNA Repair Deficiency in neurological manifestations, we will explain some of the PNKP-associated diseases.

\section{SCA3}

Spinocerebellar ataxia type 3 (SCA3) is an autosomal dominant neurodegenerative disease, and the most common inherited ataxia worldwide [25]. The disease is caused by an expansion of a polymorphic CAG trinucleotide repeat in the Ataxin 3 (ATXN3) C-terminal coding region. PNKP interacts with this protein and is inactivated by the mutant form of ATXN3, resulting in inefficient DNA repair [26]. This leads to an accumulation of DNA damage due to strand breaks with subsequent chronic activation of ATM signaling pathway.

\section{MCSZ}

Some PNKP mutations cause an autosomal recessive disease characterized by microcephaly, seizures and developmental delay and behavioral problems (denoted MCSZ). In samples of patients severely affected with MCSZ [24] (family three: homozygous E326K mutation), and mildly affected individuals (family seven: compound heterozygous for T424GfsX48 in exon 14 and a 17-bp deletion in intron 15), concentrations of PNKP protein was much lower compared with unaffected family members. This low concentration was correlated with the severely affected individuals from families 1-6. Although all these mutations impair PNKP activity, family seven compound heterozygous individuals, present a slightly 
milder phenotype, probably due to a remnant function of the enzyme. Families four and five presented the same homozygous Thr424GlyfsX48 mutation.

In mice, a hypomorphic allele that reduces the expression of PNKP is sufficient for viability but affects genome integrity [27]. The complete loss of PNKP resulted in non-viable embryos. Therefore, endogenous DNA lesions cannot be properly repaired, leading to neural cell loss and resultant neuropathology. In their experiment, Shimada and colleagues found that PNKP inactivation resulted in the loss of cerebellar neuronal population in an ATM-independent, but partially p53-dependent manner [27]. Thus, they suggested that in MCSZ, the errors in neurons associated with high oxygen consumption, depend on PNKP. According to this, it was proposed that like in other syndromes, the microcephaly in MCSZ probably implicates defective double-strand break repair (DSBR) [28]. According to this, the instability of the encoding variants of PNKP rather than specific mutations causes both microcephaly and neurodegeneration. Thus, the lack of PNKP activity would be responsible for the mutagenic effects in neurons.

The role of PNKP and DSBs in the etiology of MCSZ can be supported by the fact that the process of DSBR needs XRCC4 instead of XRCC1 (involved in SSBs). As the E236K mutation modifies the phosphatase domain, the protein is defective in being recruited to DNA damage sites, possibly through decreased interaction with XRCC4-LigIV complex after DNA damage [29]. One explanation could be that low PNKP-E326K mutant levels in MCSZ do not result from mRNA instability but cellular protein instability. However, although this specific mutation has reduced DNA kinase and DNA phosphatase functions at $37^{\circ} \mathrm{C}$, normal activity was reported at $30{ }^{\circ} \mathrm{C}$ [5]. In MCSZ, E326K mutation represents an impaired electrostatic interaction between this region and XRCC4-Lig4 due to an amino acid charge swap [30]. Interestingly, among the seven families studied with MCSZ [24], the three families that shared a homozygous mutation that resulted in the E326K change, had the most severe phenotype, suggesting a major depletion in the protein functioning.

\section{AOA4}

AOA4 is a rare autosomal neurodegenerative disorder characterized by ataxia, oculomotor apraxia, and peripheral neuropathy [3] caused by mutations in PNKP [28]. Muscle weakness progresses until most individuals start using wheelchairs by the second or third decade [30]. Interestingly, the fact that none of the mutations in MCSZ or AOA4 eliminates the phosphatase activity of the enzyme can be an indicator of lethal effect derived from a complete loss of its function [28]. This can be explained by the importance of the phosphatase activity in neurogenesis, since defects related to the 3'-phosphate end lack an effective alternative backup pathway. This lack was recently demonstrated in cancer cells lines [31]. Most of the mutations in PNKP that cause AOA4 belong to the kinase domain. Despite this, it is complicated to establish a phenotype-genotype correlation due to the compound heterozygous' clinical picture. For example, Thr424GlyfsX48 mutation is present not only in MCSZ but in AOA4 [3, 24]. In this line, another case was also reported, in which two brothers were homozygous for the p.Thr424GlyfsX48 variant in PNKP [32]. They presented a severe progressive polyneuropathy and cerebellar atrophy, microcephaly, mild epilepsy, and intellectual disability. The authors were not able to establish a definitive diagnosis since these patients presented all clinical manifestations reported for PNKP mutations. Interestingly, mice homozygous for the p.T424GfsX48 allele were lethal at the embryonal stage [27]. Compound heterozygosity involving the $\mathrm{p}$.Thr408del variant is also common in AOA4 and CMT2B2 patients [2, 3]. Recently, another research reported the case of a woman affected by AOA4 that also develop a pilocytic astrocytoma in the right cerebellar hemisphere [33]. The patient was compound heterozygous for p.T424GfsX49 / p.Tyr515ter. From the families affected by MCSZ [24], affected members of family seven were compound heterozygotes, and had the most favorable phenotype, carrying a non-coding mutation, which makes it possible to retain some PNKP activity. According to this, the phenotype generated by PNKP mutations is subject to allelic combination effects. Similar to AOA4 other neurological diseases are caused by mutations in genes that belong to the SSBR pathway. Ataxia with oculomotor apraxia-1 (AOA1) is caused by mutations in aprataxin (APTX), spinocerebellar ataxia with axonal neuropathy-1 (SCAN1) is derived from mutated Tyrosyl-DNA Phosphodiesterase 1 (TDP1). Since diseases such as AOA and SCAN1 tend to present a later onset in life, a possible explanation is that some backup mechanisms protect the cells during development [20]. These cells can even trigger apoptosis to avoid major damage in the nervous system. During neural development, progenitor cells undergo symmetric divisions that expand the size of the progenitor pool. After that, they will switch to an "asymmetric" mode of division, wherein each round produces one progenitor cell and one "postmitotic" neuron [13]. Differentiated cells can succumb to non-repaired DNA damage, but in this postmitotic scenario, apoptosis is likely to be avoided since neuronal cells are unreplaceable. Thus, as a consequence of the damage produced to this pool of cells, neurodegenerative diseases are likely to be related with DNA damage that cannot be repaired, or a progressive addition of the continuous damage of the DNA [14]. 


\section{Epileptic encephalopathies}

A group of epileptic encephalopathies has also been associated with PNKP [34, 35]. PNKP contains a C-terminal mitochondrial-targeting signal (MTS) (Fig. 1). This signal is needed for the entrance of PNKP to the mitochondria, through the mitofilin protein. This MTS site, close to the carboxy-terminus of the protein, locates at codons 432441 (ARYVQCARAA). In silico analysis indicated that mutations in this region would result in a lower affinity of the MTS "domain" because the protein failed to enter the mitochondria. Interestingly, the loss of the MTS resulted in a clear decrease in the PNKP's activity during mtDNA repair. Seizures always represent an excessive acute energy demand, irrespective of their origin in the brain [36]. The relationship between mitochondria dysfunction and seizure generation could be deduced from changes in calcium homeostasis, oxidation of ion channels and neurotransmitter transporters by ROS. Secondary mitochondrial dysfunction is a well-known mechanism in some epileptic disorders, even for those diseases that are mainly of non-mitochondrial origin [37].

Many mutations in codons that involved the MTS "domain" of PNKP are related to infantile epileptic encephalopathy and other undefined conditions (PNKP[gene] ClinVar - NCBI, 2018), and could be hypothesize that the etiology of the disease is linked to the inability of PNKP to translocate to neuronal mitochondria, or its failure to repair errors generated in the mtDNA. It has also been proposed that mitochondrial transport of $\mathrm{Ca}^{2+}$ has a role in the intracellular sequestration of this ion in neurons [38]. Due to their importance in the modulation of neuronal excitability and synaptic transmission, changes in $\mathrm{Ca}^{2+}$ homeostasis produced by errors in DNA repair (SSB), could be a direct explanation for the effects of mitochondrial dysfunction in the generation of seizures [36].

\section{Mitochondrial common etiology in axonal degeneration: using CMT as a model}

Charcot-Marie-Tooth disease (CMT) is a heterogeneous hereditary motor and sensory polyneuropathy, which is the most common hereditary neuromuscular disorder. All patterns of inheritance have been related to CMT, but autosomal-dominant inheritance is the most common. Clinically, there are demyelinating (CMT1, CMT4) axonal (CMT2) and intermediate forms of the disease [39].

Peripheral nerves need an efficient, energetic metabolism to maintain their machinery related to the transport through axons, which may be as long as $1 \mathrm{~m}$. Proper mitochondrial functioning is a critical factor for axonal and myelin formation and maintenance [39]. In fact, areas with high demands for ATP like synaptic terminals, active growth cones or axonal branches, contain more mitochondria than other cellular domains [40]. Defects in mitochondrial transport may cause local energy depletion and toxic changes in $\mathrm{Ca}^{2+}$ buffering that may trigger synaptic dysfunction [41]. The damage in the mitochondria transport system causes a similar pathology as those derived from primary mitochondrial impaired function. Both mechanisms lead to metabolic deficiencies, oxidative damage, cytotoxicity, and apoptosis [42]. This can affect the neuromuscular junction and lead to various forms of neuropathy, neurodegeneration, muscular dystrophy and paraplegia. In mature axons of cultured hippocampal neurons, an average transport velocity of approximately $0.5 \mu \mathrm{m} / \mathrm{s}$ was reported [43]. Assuming the same for motor axons, mitochondrion would reach its synaptic target in approximately 23 days [42]. Thus, the combination of distance and volume, seen from the intracellular transport system, could be the "Achilles heel" of neurons and axons [44]. In this context, we emphasize that mitochondrial transportation impairment is crucial in the process of axonal damage in CMT2 (Fig. 3a). Axonal peripheral neuropathy could also develop a secondary demyelinating phenotype. Even in that case, it is well known that proper mitochondrial functioning is a critical factor for myelin formation and maintenance [39]. The following genes have been involved in mitochondria-related axonal degeneration in CMT:

\section{KIF5A}

Kinesin Heavy Chain (KIF5A) mutations can cause axon degeneration limited to the peripheral nervous system [46]. This protein is implicated in the transport of mitochondria from the neural soma to the axon terminal. The first relationship between CMT and KIF5A was reported by [47]. These mutations were also found in Spastic paraplegia type 10 (SPG10). A single KIF5A mutation was also detected in a diagnosed CMT2 patient belonging to an SPG10 family [48]. In zebrafish, KIF5Aa mutants (heterozygous), exhibit a similar phenotype to CMT2, including the axonal degeneration. In addition, dominant homozygous mutant showed a peripheral sensory axons mitochondria deficiency and degeneration [49].

\section{GDAP1}

The Ganglioside-induced differentiation-associated protein 1 (GDAP1) is a mitochondrial fission factor mutated in CMT4 [50]. Mutations can cause perturbed axonal transport, and this may lead to impaired energy production. This process is also known in other neurodegenerative diseases due to mutations in genes related to mitochondrial dynamics [51]. Patients with recessive mutations usually have a severe axonal neuropathy with onset in early childhood. In contrast, patients with dominant GDAP1 mutations usually 


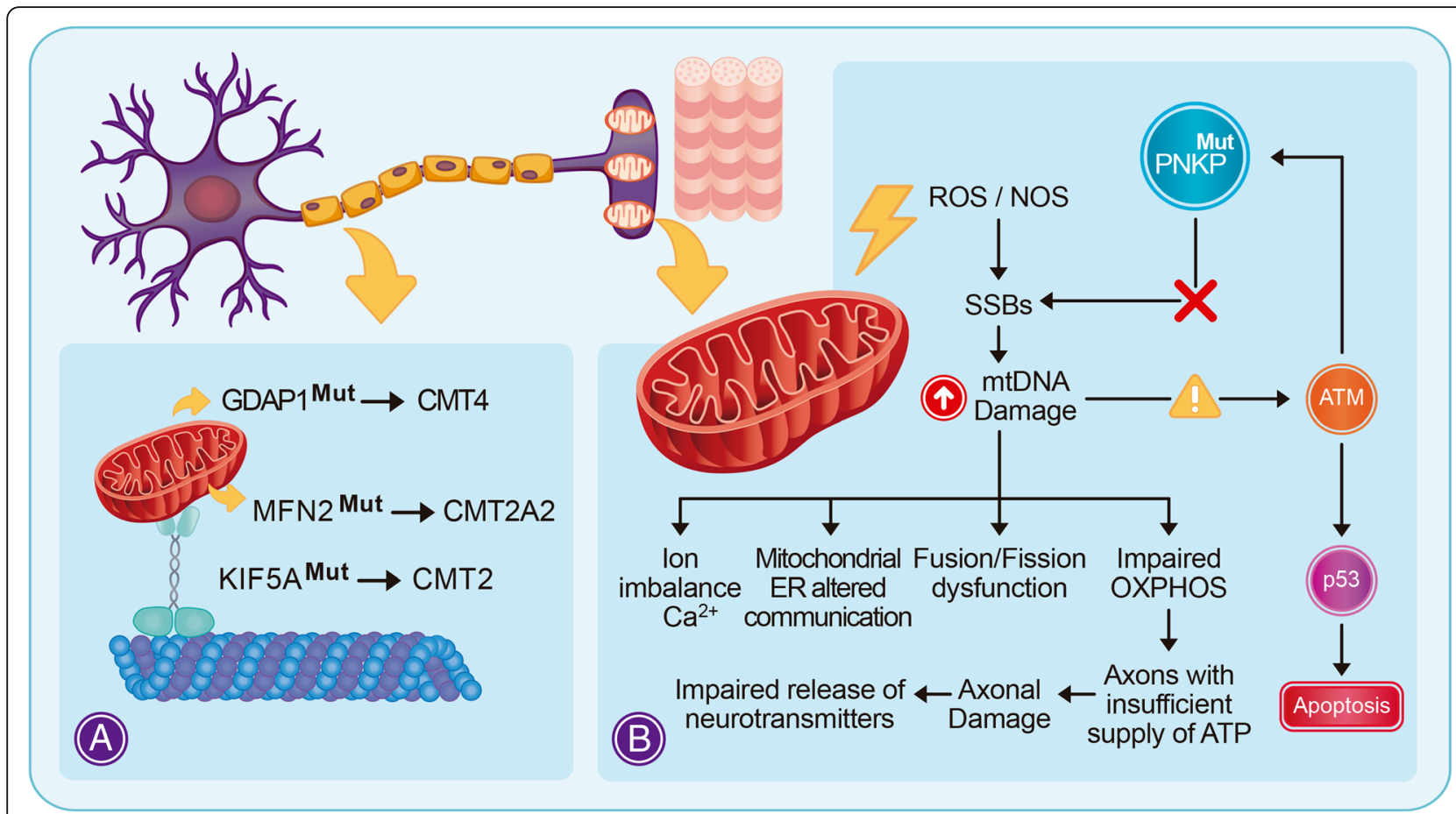

Fig. 3 Integrative representation showing the mitochondrial dysfunction as an etiology of the axonal degeneration. a Mutations associated with mitochondrial dynamics can cause axonal damage in CMT2 phenotype. $\mathbf{b}$ Role of PNKP in the maintenance of mtDNA stability. When PNKP is mutated, ROS/NOS generate an environment of genotoxic stress, that can impair the normal axonal function. Since the glycolytic capacity of neurons is restricted, mitochondrial oxidative phosphorylation is essential for neuronal ATP supply [36]. ATM-dependent phosphorylation of PNKP at serines 114 and 126 in response to oxidative DNA damage inhibits degradation of PNKP. This PNKP stability is required for DNA repair [45]. ATM can also activate p53 which undergoes apoptosis in the presence of high levels of mtDNA damage. ER: endoplasmic reticulum. OXPHOS: oxidative phosphorylation

have a mild axonal neuropathy with later onset and slower disease progression [52]. A reduced intracellular antioxidant glutathione (GHS) concentration and reduced mitochondrial membrane potential were found in patients with GDPA1 mutations [53]. Therefore, an implication of oxidative stress in the pathogenesis of the disease is suggested.

Mutations in GDAP1 can lead to mild, persistent oxidative stress in the peripheral nervous system. However, the high oxidative stress can be compensated in the unaffected central nervous system by GDAP1L1 [54]. This protein acts in response to elevated levels of oxidized glutathione, by translocating from the cytosol to mitochondria, an essential process to substitute the loss of GDAP1 expression.

\section{MFN2}

Mitofusin 2 (MFN2) is a protein from the outer mitochondria membrane that participates in mitochondrial fusion. MFN2 is also necessary for axonal mitochondrial transport [55]. In a later work, the authors suggested that although the role of MFN2 mutations in CMT2 pathogenesis is not clear, axonal degeneration could be explained due to the abnormal transport of mitochondria through the microtubule system [56]. Thus, that mechanism could explain the peripheral neuropathy and possibly the pyramidal tract involvement, where the longest axons are the first and the main ones to be affected. It is interesting that both the mutations in GDAP1 associated with mitochondrial fission and MFN2, associated with mitochondrial fusion; generate a mainly axonal CMT phenotype. The underlying cause could be deregulation in the mitochondrial dynamics, either due to the inability to be transported through the axons or due to an in-situ dysfunction caused by the disruption of mitochondrial homeostasis. This allows us to propose the hypothesis of the involvement of PNKP in the regulation of the integrity of mtDNA and the pathogenesis of CMT2. An idea that we will develop later.

There is enough evidence about pathogenic mutations that disrupt the mitochondrial homeostasis in the scenario of axonal damage. A particular case of mutations that affect mtDNA integrity is the group of mutations in the mitochondrial specific DNA polymerase (POLG). These mutations can cause an autosomal recessive ataxia-neuropathy syndrome, with onset in the first three decades of life [57]. This ataxia, named mitochondrialassociated ataxia syndrome (MIRAS), is caused by 
multiple mtDNA deletions. Symptoms include peripheral neuropathy, myoclonus, and epileptic seizures. The replication defects derived from mutations in POLG affect components of the mtDNA-encoded electron transport chain and oxidative phosphorylation [14].

The dysfunction of the mitochondrial network also has a critical role in diseases like chemotherapy-induced peripheral neuropathies (CIPN). The effects of chemotherapeutic drugs on mitochondria in the peripheral nervous system have three principal axes of impairment: calcium signaling, reactive oxygen species (ROS) and apoptosis [58]. In an experimented with the $W l d^{\mathrm{S}} \mathrm{mu}-$ tant mouse that expresses a chimeric nuclear protein Nmnatl, a protective role of this protein was shown due to an inhibition of axonal degeneration. This axonal damage was mainly caused during rotenone and vincristine treatment [59]. This suggests that this protein protects axons by reducing ROS accumulation or toxicity. Neurons treated with vincristine in the dorsal root ganglia (DRG) decreases the rate of mitochondrial dynamics, including fusion, fission, and motility; and this led to mitochondrial fragmentation and visible axon degeneration [60]. Interestingly, overexpression of an agent called cytNmnat1 inhibited the axon degeneration by preserving the normal mitochondrial dynamics. If this approach can be tested in patients affected with axonal degeneration, like CMT2B2, a possible future therapeutic target is in sight.

\section{PNKP mutations could lead to disease through mitochondrial dysfunction}

As previously mentioned, functionally active full-length PNKP is present in mitochondria as well as nuclei [69]. The idea about the contribution of mitochondria dysfunction in neurological disease is not new, not even in CMT. The contributions of the principal mutations in CMT affecting mitochondrial homeostasis have been extensively reviewed [39, 61], so the role of mitochondria in distal axonal degeneration in different peripheral neuropathies [62].

The local energy demand is known to be different between neuronal compartments, and it tends to change over time. The peripheral terminals of sensory axons represent sites of an exceptionally high ATP demand during conduction [40]. Axons consume high amounts of ATP, especially to fuel the sodium/potassium ATPase or sodium pump that functions to remove the sodium ions that enter the axon during impulse activity [63]. Because of their energy demand, most neuronal mitochondria reside in axons and dendrites [64]. As neurons cannot switch to glycolysis when oxidative phosphorylation (OXPHOS) becomes limited, mitochondrial transport, together with the dynamic processes of mitochondrial fission and fusion, facilitates the transmission of energy across long distances [65]. Whereas mitochondria play a crucial role in axonal function, it has been proposed that mtDNA is more susceptible to oxidative damage because of its lacks of protective histones [66]. Another fact is that in contrast to nDNA, almost all mtDNA is transcribed. Therefore, unrepaired mutations in the mtDNA could alter energy production and are expected to influence cell homeostasis. Thus, mtDNA genomic stability is critical for neuronal functioning [67]. The location of the mitochondria at the nerve terminals is the possible responsible for the increased susceptibility damage [68]. This vulnerable situation can be associated with age-related dysfunction and oxidative damage when compared with non-synaptic mitochondria.

In this regard, mitochondrial PNKP may play a critical role: PNKP depletion was observed to cause a significant decrease in mtDNA repair. The full restoration of repair of the $\mathrm{H}_{2} \mathrm{O}_{2}$-induced strand breaks in mitochondria required both the kinase and phosphatase activities of PNKP [69] (Fig. 3b). The authors concluded that PNKP plays a major role in the repairing of oxidized bases in the mitochondrial genome. Indeed, the major source of mtDNA lesions derives from the vast quantities of ROS produced in the mitochondria during respiration [68]. If this damage is not repaired, the mitochondria will not be able to function properly, and this can lead to neuropathy.

In the mitochondria, the base excision repair (BER) and SBBR pathways are the primary DNA repair mechanisms [70]. In this line, another work demonstrated that PNKP-depleted human mitochondrial extracts showed a significant decrease in both BER and SSBR activities. In fact, PNKP was found to be the major 3-phosphatase within mitochondria [71]. Various mtDNA lesions, including point mutations and large-scale deletions of mtDNA, can lead to mitochondrial dysfunction and cellular apoptosis [20]. In fact, mtDNA oxidative stress induces strand breaks with a much higher frequency than mutagenic lesions [72]. These lesions represent an impairment for both replication and transcription of mtDNA. When the damage rises above a threshold level, it can induce degradation of mtDNA molecules [73]. Future clinical research should also analyze the mtDNA damage in DNA repair deficiency, as it could be useful as a biomarker in patients with mutations in PNKP and other enzymes from the SSBR pathway.

\section{Mitochondrial dysfunction as a common signature in cerebellar degeneration}

Cerebellar degeneration is a common neurological feature in ataxia. An interesting fact about the cerebellum is its extensive development after birth. During this phase, replication stress is predictable due to the rapid cell proliferation, leading to more DNA breaks than in other parts of the nervous system [67]. The cerebellar degeneration that leads to the ataxia presenting in 
patients with mitochondrial dysfunction can be primary (caused by affecting the cerebellum and its connections), or secondary (due to loss of proprioception derived from peripheral neuropathy or neuronopathy, or a combination of both) like in spinocerebellar ataxia [74].

Cerebellar development starts with the neurogenesis of Purkinje cells (PC) around embryonic day E10.5. Their proliferation ceases shortly before birth, but in the postnatal period, they still grow, mature and develop their synaptic network [75]. Purkinje neurons possess vast numbers of synaptic connections via their dendritic tree, which implies a high-energy demand for the integration of excitatory and inhibitory synaptic inputs and subsequent maintenance of ion gradients. One abnormality in PC mitochondria is observed in the pcd mouse, a recessive model of neurodegeneration, involving cerebellum and retina. It is derived from mutations in the Agtbp1 gene (Nna1), which expresses a protein that translocates to mitochondria. Agtbp1 loss of function results in altered bioenergetics and mitochondrial dysfunction. As a result, mice present progressive gait ataxia [76]. Following this idea, we summarize several genes that are involved in this pathogenic mechanism based on mitochondrial-related cerebellar degeneration.

\section{Sacs}

Autosomal recessive spastic ataxia of Charlevoix-Saguenay (ARSACS) is a childhood-onset neurological disease characterized by cerebellar ataxia with spasticity, pyramidal syndrome and peripheral neuropathy. It is caused by mutations in the SACS gene encoding sacsin. The knockout mice, display age-dependent neurodegeneration of cerebellar PC due to impaired mitochondrial network and the accumulation of mitochondria in the soma and proximal dendrites [77].

\section{LIG iii}

The inactivation of Lig3 in the mouse nervous system results in mtDNA loss leading to profound mitochondrial dysfunction, disruption of cellular homeostasis and incapacitating ataxia [78]. Morphological abnormalities of the mitochondria at postnatal times coincided with the emergence of ataxia. This was demonstrated in cerebellar PC neurons that showed distorted mitochondrial cristae structure and broad changes in its morphology. There was also a marked reduction of complex III and IV immunostaining.

\section{APTX}

Although cytoplasmic aprataxin may be present at a low level in all cell types, it seems to be more predominant in neuron and neuron-like cell lines and tissues [73]. The expression of the total aprataxin transcript and aprataxin MTS-encoding transcript varies among the different brain regions but were more abundant in the cerebellum. Aprataxin knockdown had a significantly higher level of ROS than control cells, lower citrate synthase activity, and reduced mtDNA copy number [73]. As the protein has different isoforms (differing from each other at the $\mathrm{N}$ - and $\mathrm{C}$ - termini), not every isoform has the MTS sequence.

\section{TDP1}

TDP1 hydrolyzes the phosphodiester bond at a DNA 3 '-end linked to a tyrosyl moiety and is involved in the repair of topoisomerase I (Top1) DNA covalent complexes [79]. A fraction of the TDP1 encoded by the nuclear gene translocates to mitochondria [82]. As mitochondrial base excision repair depends on TDP1 activity, it is required for efficient repair of oxidative damage in that organelle [80-82].

Cerebellar neurons and primary astrocytes derived from Tdp1-/- mice present an inability to rapidly repair DNA SSBs associated with Top1-DNA complexes or oxidative damage [83]. Moreover, loss of the protein resulted in age-dependent and progressive cerebellar atrophy in spinocerebellar ataxia with axonal neuropathy-1 (SCAN1) patients. The overexpression of a toxic form of mitochondrial topoisomerase I (TOP1mt*) generates excessive mitochondrial protein-linked DNA breaks (mtPDBs), that results in a TDP1-dependent compensatory upregulation of mitochondrial gene transcription. Misassembled of the ETC complex III resulted in the absence of TDP1 due to the imbalance in transcription of mitochondrial- and nuclear-encoded electron transport chain (ETC) subunits. Bioenergetics profiling further reveals that TDP1 promotes oxidative phosphorylation under both basal and high-energy demands [84].

\section{ATXN1}

In cerebellar tissue of a Purkinje cell-driven spinocerebellar ataxia type 1 (SCA1), mice display deficits in cerebellar OXPHOS complex I (NADH-coenzyme Q oxidoreductase) [85]. In SCA1, Complex I genes are upregulated at the time of onset and upregulation persists into the late-stage disease.

SCA1 transgenic mice present clinical features of cerebellar dysfunction. The loss of PC is evident in both heterozygous and homozygous six-month-old mice. However, apoptosis does not seem to be involved in PC degeneration. While levels of brain-derived mtDNA are not different between SCA1 and control mice, mtDNA levels are significantly reduced in cerebellum of SCA1 mice [86].

\section{ATXN3}

In SCA3, the interaction of PNKP with mutant ATXN3 markedly abrogate PNKP's enzymatic activity and DNA 
repair efficacy, resulting in persistent accumulation of strand breaks. Mutant ATXN3 potently activates the DNA damage-response ATM signaling pathway [26]. In SCA3, complex II exhibits a consistent tendency toward decreased activity in the presence of expanded ataxin-3, particularly in differentiated PC [87]. In neuronal cultures of the cerebellum, striatum and substantia nigra, the polyglutamine-expanded ataxin-3 (Q79) activates mitochondrial apoptotic pathway and leads to neuronal death by upregulating Bax expression and downregulating $\mathrm{Bcl}-\mathrm{xL}$ expression of cerebellar, striatal or substantia nigra neurons [88].

The presence of the full-length mutant ataxin-3 in Q71 homozygotes is either positive in cerebellum and over the cerebral cortex. However, the fragment and aggregate of ATXN3 were detected more abundantly in the cerebellum [89]. Truncated mutant ATXN3 led to more mitochondrial fission due to the lower expression of Mfn-1 and Mfn-2. In transgenic mouse models, truncated mutant ATXN3 also led to mitochondrial dysfunction, neurodegeneration and cell death in the cerebellum [90].

\section{POLG}

In POLG-related neurodegeneration, the primary consequence of POLG mutation in neurons is mtDNA depletion. Progressive accumulation of mtDNA deletions and point mutations was found accompanied by increasing numbers of complex I-deficient neurons [91]. Severe Purkinje and granule cell loss and Bergmann gliosis in some patients characterized these lesions.

\section{FXN}

Friedreich's ataxia is an autosomal recessive inherited neurodegenerative disorder, caused by an expansion mutation within intron 1 of the FXN gene. It is characterized by progressive spinocerebellar ataxia, cardiomyopathy, scoliosis, and diabetes. The frataxindeficient YG8R mouse model showed a limitation of the maintenance of mitochondrial membrane potential in cerebellar granule neurons. Specifically, a deficiency in Complex I increased levels of mitochondrial and cytosolic ROS. Excessive ROS production results in oxidative stress and diminishes the level of glutathione (GSH) [92]. Deficient expression of frataxin leads to deleterious alterations in iron metabolism in the mitochondrial matrix that is presumed to play a crucial role in the oxidative damage and subsequent degenerative features of this disease [93].

Despite the known role of mitochondria dysfunction in cerebellar degeneration that usually leads to ataxia, it is not clear why diseases such as POLG and Friedreich's ataxia occur with many systemic clinical manifestations. Interestingly, ataxia associated with mitochondrial dysfunction seems to be the primary clinical manifestation, if caused by mutations in the enzymes of the SSBR pathway.

\section{ATM}

Ataxia-telangiectasia (AT) has been the archetypal neurological disorder associated with DNA double-strand break repair, mainly because of its predisposition to cancer [94]. The disease has an extended phenotype, as a cause of the dual function of ATM: DNA repair and regulation of oxidative stress and mitochondrial homeostasis. Although AT presents defects in DNA repair and mitochondrial dysfunction, the clinical manifestations have a systemic pattern. Thus, the pathological role of DNA repair deficiency in AT seems to extend to all cells in the body, which explains the predisposition to cancer, something rarely seen in other types of ataxia. In contrast, the mitochondrial dysfunction seems to be more crucial in neurons, especially in the cerebellum as recently demonstrated [95-97] (Fig. 4).

In some other the diseases caused by mutations in DNA repair enzymes, there was no clarity regarding the possible mitochondrial involvement. Recently, in Xeroderma pigmentosum A (XPA), a disease characterized by dermatological ailments but also progressive cerebral and cerebellar atrophy, sensorineural hearing loss and neuropathy, mitochondrial dysfunction was shown. The dysfunction seems to originate from defects in mitophagy, possibly mediated by hyperactivation of PARP1 [98-100]. Another interesting case is a recent report of a patient with mutations in XRCC1 who presented motor ocular apraxia, axonal neuropathy, and progressive cerebellar ataxia [101]. In this case, the authors also determined that hyperactivation of PARP1 was the cause of cerebellar ataxia. To understand the implication of this pathway in the mitochondrial dysfunction, it is also important to examine the PARP1/NAD ${ }^{+} / \mathrm{SIRT} 1$ axis. When PARP1 is activated, it orchestrates DNA repair, which leads to the loss of $\mathrm{NAD}^{+}$and acetyl-CoA. This results in inhibition of SIRT1 that leads to an increase in mitochondrial ROS, owing to decreased activation of stress response factors such as AMPK, FOXOs, and PGC1 $\alpha$. Also, SIRT1 has been shown to regulate several DNA repair pathways positively; including xeroderma pigmentosum group A-complementing protein (XPA), KU70, ataxia telangiectasia mutated (ATM) and thymine DNA glycosylase (TDG) [102]. In this regard, PARP1 appears as a common potential therapeutic target for mitochondrial dysfunction in DNA repair disorders.

\section{Principles of DNA repair deficiency disorders}

DNA repair deficiencies (DRD) result from different mutations in DNA repair enzymes. There are critical considerations to understand the molecular basis of several 


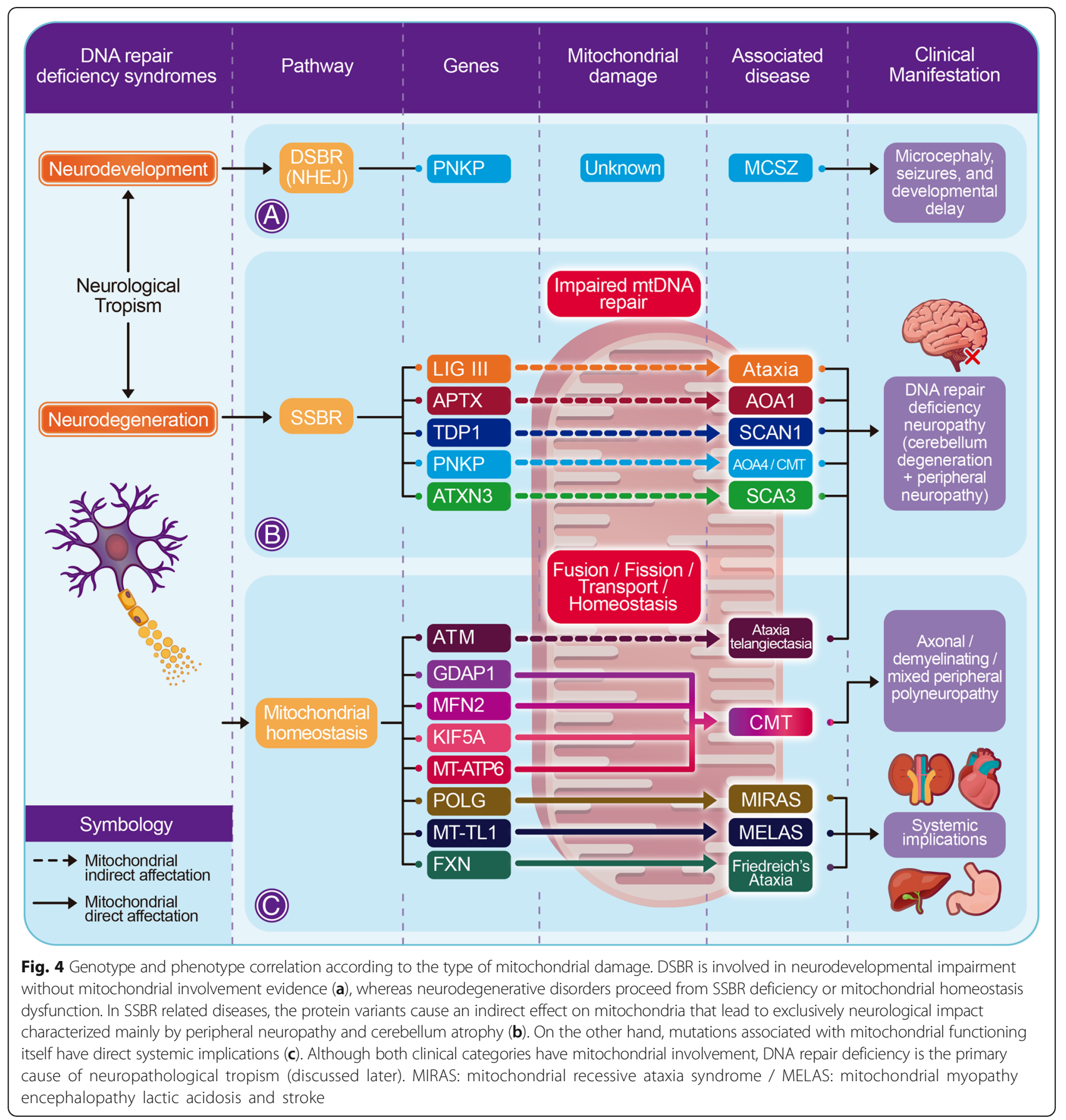

neurological diseases, and therefore, for establishing the correlation between the genotype and phenotype. From the available data, here we summarize the interactions of a group of elements that combined, can explain the molecular pathogenesis of these diseases.

The first consideration that should be taken into account is whether a pathological tropism (PT) is observed in the disease. PT can be understood as the tendency of a disease to affect a particular tissue. In the case of the
SSBR pathway, it would mean an exclusive neurological effect [21]. This is possibly explained by the vulnerabilities of the nervous system regarding higher oxidative damage and higher dependence on DNA repair, compared to the rest of the body.

DRD neurological disorders can be divided into those affecting the neurons during development and the ones that affect the mature nervous system. In the process of neurogenesis, the replication stress associated with high 
rates of proliferation is a major source of DNA damage that can perturb neural development. Indeed, it can be the main etiology involved in neurodevelopmental disease. On the other hand, in the mature nervous system (post-mitotic cells) the high transcriptional activity associated with metabolism result in cell dysfunction and possibly death.

The next consideration to comprehend the specific pathology related to a mutation is the momentum, which is the stage of cellular development when the damage occurs. In the case of neurons, it can be mitotic or post-mitotic. Neurodevelopment-associated damage is more likely to originate in the mitotic momentum, and neurodegenerative diseases would begin principally post-mitotic. This is relevant to understand and classify the pathologies of the central nervous system. We suggest a more in-depth evaluation of the categories established for DRD disorders, especially those that appear to arise in the development but has neurodegenerative implications. Evidence indicates that AOA causative mutations affect the development of Purkinje cells through the impairment of mitochondrial homeostasis. Nevertheless, clinical manifestations occur in the first decade and progress through life, showing a neurodegenerative pathology. Momentum matters, since the most common types of DNA damage in the nervous system, are different at both stages, just like the repair systems available. In developing cells, the most important source of errors is the DNA replication itself. As we explained before, in post-mitotic neurons, the main source of mutations is the stress associated with transcription and the high-energy demand, in which the mitochondria are involved, primarily due to the ROS production [68]. This is what we define as the source of mutagenic damage.

The damage derived from mitotic cells is predominately double-strand breaks (DSBs), normally repaired by homologous recombination and the NHEJ (PNKP $+\mathrm{XRCC} 4$ complex). If breaks cannot be repaired, it would be lethal at this stage through apoptosis. In post-mitotic neurons, non-homologous end joining (NHEJ) is the sole pathway available to prevent accumulation of DNA double-strand breaks [103]. On the other hand, SSBs represent a serious threat to genetic stability and cell survival, especially because neurons have low levels of antioxidant enzymes and high metabolic activity. Proliferative cells become increasingly dependent on SSBR, and post-mitotic cells depend on SSBR exclusively, in which PNKP has a crucial role, especially in mitochondria-derived oxidative stress damage $[11,13]$. In mature cells, the high frequency of error linked to SSBs and the lack of alternative pathways, especially in the mitochondria, could lead to an accumulation of damage. This may explain the late onset and slow progression of neurodegenerative diseases. Therefore, depending on the momentum and source, it is possible to classify the specific type of error in DNA: this can be defined as the principle of the (single or double) affected strand.

As observed for PNKP, XRCC1 is required in the neurogenesis of cerebellar interneuron [104]. XRCC1 deficiency drives a progressive and persistent accumulation of strand breaks in mature neuronal populations. According to this, it is possible to notice an overlap between the phenotype caused by mutant variants from the same pathway (SSBR), especially in the impaired development of the cerebellum. It could be hypothesized that the clinical spectrum can also be related to the level of disruption in the SSBR cascade.

Since PNKP could share the same mutations in both neurodevelopmental and neurodegenerative diseases, the problem cannot be referred to the protein variant itself, but how the PNKP mutant interacts with the available repair machinery for a specific momentum and strand. This can be synthesized as the effect of the mutation in the enzyme and the particular pathway involved that ultimately leads to a specific phenotype. In tissues like the cerebellum where mutations arise with a very high rate (due to the high proliferation rate), and axons, where the energy demand is so high that it compromises mitochondrial homeostasis, mutations in DNA repair enzymes could lead to the observed neurodegenerative phenotype. Thus, at this point, it is possible to integrate the mitochondrial dysfunction in both axonal and cerebellar degeneration and introduce the last principle or consideration: mitochondrial intervention.

Since the energetic requirement of neurons is very high, axons contain more mitochondria than other cellular domains. This mitochondrial activity generates a high amount of ROS with the subsequent SSBs. The cerebellum cells, such as PC, have a high rate of postnatal replication, which is highly dependent on mitochondrial activity. Most diseases with ataxia as primary clinical manifestation due to mutations in DNA repair enzymes (SSBR) present an indirect effect of mitochondrial function (Fig. 4b). Therefore, although the primary damage is more likely to derive from unrepaired nuclear DNA damage (associated with replication stress), there is a concomitant role between nuclear and mitochondrial genome dysfunction as the underlying pathogenic process. According to this, we propose a model in which mutations in DNA repair enzymes such as PNKP, APTX, TDP1, ATXN1/3, and others, cause an indirect effect on mitochondria that lead to exclusively neurological impact due to the specific metabolic and genome maintenance characteristics of the central nervous system. This damage is mainly peripheral neuropathy and cerebellum atrophy, showing an evident mitochondrial SSBR-dependent dysfunction (Fig. 4b). To determine the moment in which the mitochondrial damage occurs could be important for a future therapeutic approach. 
Diseases such as Friedreich's ataxia and MIRAS (caused by POLG mutations) present an extended phenotype, maybe derived from the equal vulnerability that these variants confer to the mitochondria in the whole organism. Even though the clinical difference is evident, still more research is needed to understand the molecular basis of this systemic damage compared with the diseases that tend to present a pathological tropism. One idea is that mtDNA often shows an uneven distribution between cells of the same tissue, so this can explain why defects appear only above a mutation-specific threshold in vulnerable organs. This can generate a mosaic pattern of mitochondrial dysfunction and differences in the distribution patterns of the pathogenic mutations can be observed and be clinically relevant [36].

\section{Conclusions}

Deficiency in DNA repair can lead to multiorgan diseases or can be confined to the nervous system. Enzymes such as PNKP, TDP1, and APTX, are not only active in the nucleus of neurons but also in their mitochondria. They constitute an example of how the deficiency in the DNA SSBR machinery converges with mitochondrial dysfunction, in the etiology of pathologies with an exclusive neurological involvement. There is enough evidence to conclude that mitochondrial dysfunction is what best explains the clinical manifestations and progression in neurodegeneration. Therefore, intensive research should be directed to develop new therapeutic strategies, targeting the common feature of these diseases: the mitochondria.

\section{Abbreviations}

AOA: Ataxia-oculomotor apraxia; APTX: Aprataxin; ATM: Ataxia-telangiectasia mutated; ATXN3: Ataxin 3; CIPN: Chemotherapy-induced peripheral neuropathies; CMT: Charcot-Marie-Tooth; DDR: DNA repair deficiency; DSBR: Double-strand break repair; DSBs: Double-strand breaks; FXN: Frataxin; GDAP1: Ganglioside-induced differentiation-associated protein 1. GHS: Glutathione; KIF5A: Kinesin Heavy Chain 5A; MCSZ: Microcephaly and Seizures; MELAS: Mitochondrial myopathy encephalopathy lactic acidosis and stroke; MFN2: Mitofusin 2; MIRAS: Mitochondrial-associated ataxia syndrome; mtDNA: Mitochondrial DNA; MTS: Mitochondrial-targeting signal; nDNA: Nuclear DNA; NHEJ: Non-homologous end joining; PC: Purkinje cell; PNKP: Polynucleotide kinase 3'-phosphatase; POLG: Polymerase $\gamma$; ROS: Reactive oxygen species; SCA3: Spinocerebellar ataxia type 3; SCAN1: Spinocerebellar ataxia with axonal neuropathy-1; SPG10: Spastic paraplegia type 10; SSBR: Single strand breaks repair; SSBs: Single strand breaks; TDP1: Tyrosyl-DNA Phosphodiesterase 1; XRCC1/4: X-Ray Repair Cross Complementing $1 / 4$

\section{Acknowledgments}

We thank Gabriel Jiménez Huezo for the graphic design of the pictures in this paper.

\section{Funding}

Support from the University of Costa Rica is gratefully acknowledged (project 111-B2-372).

Availability of data and materials Not applicable.

\section{Authors' contributions}

LBG drafted the manuscript; $A$ L and LBG performed constructive outline and critical editing. Authors read and approved the final manuscript.

Ethics approval and consent to participate

Not applicable.

\section{Consent for publication}

All authors consent for publication.

\section{Competing interests}

The authors declare that they have no competing interests.

Received: 7 February 2019 Accepted: 24 April 2019

Published online: 09 May 2019

\section{References}

1. Ciccia A, Elledge SJ. The DNA damage response: making it safe to play with knives. Mol Cell. 2010;40:179-204.

2. Leal A, Bogantes-Ledezma S, Ekici AB, Uebe S, Thiel $C T$, Sticht $H$, et al. The polynucleotide kinase 3'-phosphatase gene (PNKP) is involved in CharcotMarie-tooth disease (CMT2B2) previously related to MED25. Neurogenetics. 2018;19:215-25.

3. Bras J, Alonso I, Barbot C, Costa MM, Darwent L, Orme T, et al. Mutations in PNKP cause recessive Ataxia with oculomotor apraxia type 4. Am J Hum Genet. 2015:96:474-9.

4. Breslin C, Caldecott KW. DNA 3'-phosphatase activity is critical for rapid global rates of single-Strand break repair following oxidative stress. Mol Cell Biol. 2009;29:4653-62.

5. Reynolds JJ, Walker AK, Gilmore EC, Walsh CA, Caldecott KW. Impact of PNKP mutations associated with microcephaly, seizures and developmental delay on enzyme activity and DNA strand break repair. Nucleic Acids Res. 2012:40:6608-19.

6. Weinfeld M, Mani RS, Abdou I, Aceytuno RD, Glover JNM. Tidying up loose ends: the role of polynucleotide kinase/phosphatase in DNA strand break repair. Trends Biochem Sci. 2011;36:262-71.

7. Della-Maria J, Hegde ML, McNeill DR, Matsumoto Y, Tsai M-S, Ellenberger $T$, et al. The interaction between polynucleotide kinase phosphatase and the DNA repair protein XRCC1 is critical for repair of DNA alkylation damage and stable association at DNA damage sites. J Biol Chem. 2012;287:39233-44.

8. Bernstein NK, Williams RS, Rakovszky ML, Cui D, Green R, Karimi-Busheri F, et al. The molecular architecture of the mammalian DNA repair enzyme, polynucleotide kinase. Mol Cell. 2005;17:657-70.

9. Audebert M, Salles B, Weinfeld M, Calsou P. Involvement of polynucleotide kinase in a poly(ADP-ribose) Polymerase-1-dependent DNA double-strand breaks rejoining pathway. J Mol Biol. 2006;356:257-65.

10. Barzilai A. The contribution of the DNA damage response to neuronal viability. Antioxid Redox Signal. 2007;9:211-8.

11. Caldecott KW. Single-strand break repair and genetic disease. Nat Rev Genet. 2008;9:619-31.

12. Tann AW, Boldogh I, Meiss G, Qian W, Van Houten B, Mitra S, et al. Apoptosis induced by persistent single-strand breaks in mitochondrial genome: Critical role of exog (5'-Exo/Endonuclease) in their repair. J Biol Chem. 2011;286:31975-83.

13. Narciso L, Parlanti E, Racaniello M, Simonelli V, Cardinale A, Merlo D, et al. The response to oxidative DNA damage in neurons: mechanisms and disease. Neural Plast. 2016;2016:1-14.

14. McKinnon PJ. Maintaining genome stability in the nervous system. Nat Neurosci. 2013;16:1523-9.

15. Fortini P, Ferretti C, Dogliotti E. The response to DNA damage during differentiation: pathways and consequences. Mutat Res Mol Mech Mutagen. 2013:743-744:160-8.

16. Fortini P, Dogliotti E. Mechanisms of dealing with DNA damage in terminally differentiated cells. Mutat Res Mol Mech Mutagen. 2010;685:38-44.

17. Suberbielle E, Sanchez PE, Kravitz AV, Wang X, Ho K, Eilertson K, et al. Physiologic brain activity causes DNA double-strand breaks in neurons, with exacerbation by amyloid- $\beta$. Nat Neurosci. 2013;16:613-21.

18. Lieber MR, Ma Y, Pannicke U, Schwarz K. Mechanism and regulation of human non-homologous DNA end-joining. Nat Rev Mol Cell Biol. 2003;4: $712-20$. 
19. Frank KM, Sharpless NE, Gao Y, Sekiguchi JM, Ferguson DO, Zhu C, et al. DNA ligase IV deficiency in mice leads to defective neurogenesis and embryonic lethality via the p53 pathway. Mol Cell. 2000;6:993-1002.

20. McKinnon PJ. DNA repair deficiency and neurological disease. Nat Rev Neurosci. 2009;10:100-12.

21. McKinnon PJ. Genome integrity and disease prevention in the nervous system. Genes Dev. 2017;31:1180-94.

22. Caldecott KW. DNA single-strand breaks and neurodegeneration. DNA Repair. 2004;3:875-82.

23. Madabhushi R, Pan L, Tsai L-H. DNA damage and its links to neurodegeneration. Neuron. 2014;83:266-82.

24. Shen J, Gilmore EC, Marshall CA, Haddadin M, Reynolds JJ, Eyaid W, et al. Mutations in PNKP cause microcephaly, seizures and defects in DNA repair. Nat Genet. 2010;42:245-9.

25. Ruano L, Melo C, Silva MC, Coutinho P. The global epidemiology of hereditary Ataxia and spastic paraplegia: a systematic review of prevalence studies. Neuroepidemiology. 2014;42:174-83.

26. Gao R, Liu Y, Silva-Fernandes A, Fang X, Paulucci-Holthauzen A, Chatterjee $A$, et al. Inactivation of PNKP by mutant ATXN3 triggers apoptosis by activating the DNA damage-response pathway in SCA3. PLoS Genet. 2015: 11:e1004834 Pearson CE, editor.

27. Shimada M, Dumitrache LC, Russell HR, McKinnon PJ. Polynucleotide kinasephosphatase enables neurogenesis via multiple DNA repair pathways to maintain genome stability. EMBO J. 2015;34:2465-80.

28. Dumitrache LC, McKinnon PJ. Polynucleotide kinase-phosphatase (PNKP) mutations and neurologic disease. Mech Ageing Dev. 2017;161:121-9.

29. Aceytuno RD, Piett CG, Havali-Shahriari Z, Edwards RA, Rey M, Ye R, et al. Structural and functional characterization of the PNKP-XRCC4-LigIV DNA repair complex. Nucleic Acids Res. 2017;45:6238-51.

30. Jiang B, Glover JNM, Weinfeld M. Neurological disorders associated with DNA strand-break processing enzymes. Mech Ageing Dev. 2017;161:130-40.

31. Chalasani SL, Kawale AS, Akopiants K, Yu Y, Fanta M, Weinfeld M, et al. Persistent 3'-phosphate termini and increased cytotoxicity of radiomimetic DNA double-strand breaks in cells lacking polynucleotide kinase/phosphatase despite presence of an alternative 3'-phosphatase. DNA Repair. 2018;68:12-24.

32. Poulton C, Oegema R, Heijsman D, Hoogeboom J, Schot R, Stroink H, et al. Progressive cerebellar atrophy and polyneuropathy: expanding the spectrum of PNKP mutations. Neurogenetics. 2013;14:43-51.

33. Scholz C, Golas MM, Weber RG, Hartmann C, Lehmann U, Sahm F, et al. Rare compound heterozygous variants in PNKP identified by whole exome sequencing in a German patient with ataxia-oculomotor apraxia 4 and pilocytic astrocytoma. Clin Genet. 2018;94:185-6.

34. Carvill GL, Heavin SB, Yendle SC, McMahon JM, O'Roak BJ, Cook J, et al. Targeted resequencing in epileptic encephalopathies identifies de novo mutations in CHD2 and SYNGAP1. Nat Genet. 2013:45:825-30.

35. Mastrangelo M, Leuzzi V. Genes of early-onset epileptic encephalopathies: from genotype to phenotype. Pediatr Neurol. 2012;46:24-31.

36. Zsurka G, Kunz WS. Mitochondrial dysfunction and seizures: the neuronal energy crisis. Lancet Neurol. 2015;14:956-66.

37. Bindoff LA, Engelsen BA. Mitochondrial diseases and epilepsy: mitochondrial diseases and epilepsy. Epilepsia. 2012;53:92-7.

38. Sanganahalli BG, Herman P, Hyder F, Kannurpatti SS. Mitochondrial calcium uptake capacity modulates neocortical excitability. J Cereb Blood Flow Metab. 2013;33:1115-26

39. Pareyson D, Saveri P, Sagnelli A, Piscosquito G. Mitochondrial dynamics and inherited peripheral nerve diseases. Neurosci Lett. 2015;596:66-77.

40. Sajic M, Mastrolia V, Lee CY, Trigo D, Sadeghian M, Mosley AJ, et al. Impulse conduction increases mitochondrial transport in adult mammalian peripheral nerves in vivo. PLoS Biol. 2013;11:e1001754 Barres BA, editor.

41. Sheng Z-H, Cai Q. Mitochondrial transport in neurons: impact on synaptic homeostasis and neurodegeneration. Nat Rev Neurosci. 2012;13:77-93.

42. Zinsmaier KE, Babic M, Russo GJ. Mitochondrial Transport Dynamics in Axons and Dendrites. In: Koenig E, editor. Cell Biol Axon. Berlin: Springer Berlin Heidelberg; 2009. p. 361-81. Available from: http://link.springer.com/ 10.1007/400_2009_20. [cited 2019 Jan 19].

43. Ligon LA, Steward O. Movement of mitochondria in the axons and dendrites of cultured hippocampal neurons. J Comp Neurol. 2000;3:340-50.

44. Goldstein LSB. Kinesin molecular motors: transport pathways, receptors, and human disease. Proc Natl Acad Sci. 2001;98:6999-7003.

45. Parsons JL, Khoronenkova SV, Dianova II, Ternette N, Kessler BM, Datta PK, et al. Phosphorylation of PNKP by ATM prevents its proteasomal degradation and enhances resistance to oxidative stress. Nucleic Acids Res. 2012;40: 11404-15.

46. Nam DE, Yoo DH, Choi SS, Choi B-O, Chung KW. Wide phenotypic spectrum in axonal Charcot-Marie-tooth neuropathy type 2 patients with KIF5A mutations. Genes Genomics. 2018;40:77-84.

47. Goizet C, Boukhris A, Mundwiller E, Tallaksen C, Forlani S, Toutain A, et al. Complicated forms of autosomal dominant hereditary spastic paraplegia are frequent in SPG10. Hum Mutat. 2009;30:E376-85.

48. Crimella C, Baschirotto C, Arnoldi A, Tonelli A, Tenderini E, Airoldi G, et al. Mutations in the motor and stalk domains of KIF5A in spastic paraplegia type 10 and in axonal Charcot-Marie-tooth type 2. Clin Genet. 2012;82:157-64.

49. Campbell PD, Shen K, Sapio MR, Glenn TD, Talbot WS, Marlow FL. Unique function of kinesin Kif5A in localization of mitochondria in axons. J Neurosci. 2014:34:14717-32.

50. Pareyson D, Marchesi C. Diagnosis, natural history, and management of Charcot-Marie-tooth disease. Lancet Neurol. 2009;8:654-67.

51. Cassereau J, Chevrollier A, Gueguen N, Desquiret V, Verny C, Nicolas G, et al. Mitochondrial dysfunction and pathophysiology of Charcot-Marie-tooth disease involving GDAP1 mutations. Exp Neurol. 2011;227:31-41.

52. Timmerman V, Clowes VE, Reid E. Overlapping molecular pathological themes link Charcot-Marie-tooth neuropathies and hereditary spastic paraplegias. Exp Neurol. 2013;246:14-25.

53. Noack R, Frede S, Albrecht P, Henke N, Pfeiffer A, Knoll K, et al. CharcotMarie-tooth disease CMT4A: GDAP1 increases cellular glutathione and the mitochondrial membrane potential. Hum Mol Genet. 2012;21:150-62.

54. Niemann A, Huber N, Wagner KM, Somandin C, Horn M, Lebrun-Julien F, et al. The Gdap1 knockout mouse mechanistically links redox control to Charcot-Marie-tooth disease. Brain. 2014;137:668-82.

55. Misko A, Jiang S, Wegorzewska I, Milbrandt J, Baloh RH. Mitofusin 2 is necessary for transport of axonal mitochondria and interacts with the Miro/ Milton complex. J Neurosci. 2010;30:4232-40.

56. Misko AL, Sasaki Y, Tuck E, Milbrandt J, Baloh RH. Mitofusin2 mutations disrupt axonal mitochondrial positioning and promote axon degeneration. J Neurosci. 2012;32:4145-55

57. Longley MJ, Graziewicz MA, Bienstock RJ, Copeland WC. Consequences of mutations in human DNA polymerase $\gamma$. Gene. 2005;354:125-31.

58. Canta A, Pozzi E, Carozzi V. Mitochondrial dysfunction in chemotherapyinduced peripheral neuropathy (CIPN). Toxics. 2015;3:198-223.

59. Press C, Milbrandt J. Nmnat delays axonal degeneration caused by mitochondrial and oxidative stress. J Neurosci. 2008;28:4861-71.

60. Berbusse GW, Woods LC, Vohra BPS, Naylor K. Mitochondrial dynamics decrease prior to axon degeneration induced by vincristine and are partially rescued by overexpressed cytNmnat1. Front Cell Neurosci. 2016;10 Available from: http://journal.frontiersin.org/Article/10.3389/fncel.2016.00179/abstract. [cited 2019 Jan 19].

61. Palau F, Estela A, Pla-Martín D, Sánchez-Piris M. The role of mitochondrial network dynamics in the pathogenesis of Charcot-Marie-tooth disease. Inherit Neuromuscul dis Transl Pathomechanisms Ther. 6th ed. Dordrecht: Springer Science+Business Media B.V; 2009. p. 129-37.

62. Cashman CR, Höke A. Mechanisms of distal axonal degeneration in peripheral neuropathies. Neurosci Lett. 2015;596:33-50.

63. Mao P, Reddy PH. Is multiple sclerosis a mitochondrial disease? Biochim Biophys Acta (BBA) - Mol Basis Dis. 2010;1802:66-79.

64. Ashrafi G, Schlehe JS, LaVoie MJ, Schwarz TL. Mitophagy of damaged mitochondria occurs locally in distal neuronal axons and requires PINK1 and Parkin. J Cell Biol. 2014;206:655-70.

65. Knott AB, Perkins G, Schwarzenbacher R, Bossy-Wetzel E. Mitochondrial fragmentation in neurodegeneration. Nat Rev Neurosci. 2008;9:505-18.

66. Richter C, Park JW, Ames BN. Normal oxidative damage to mitochondrial and nuclear DNA is extensive. Proc Natl Acad Sci U S A. 1988;85:6465-7.

67. El-Khamisy SF. To live or to die: a matter of processing damaged DNA termini in neurons: DNA end processing and neurodegeneration. EMBO Mol Med. 2011;3:78-88.

68. Grimm A, Eckert A. Brain aging and neurodegeneration: from a mitochondrial point of view. J Neurochem. 2017;143:418-31.

69. Tahbaz N, Subedi S, Weinfeld M. Role of polynucleotide kinase/ phosphatase in mitochondrial DNA repair. Nucleic Acids Res. 2012;40: 3484-95.

70. Kazak L, Reyes A, Holt IJ. Minimizing the damage: repair pathways keep mitochondrial DNA intact. Nat Rev Mol Cell Biol. 2012;13:659-71. 
71. Mandal SM, Hegde ML, Chatterjee A, Hegde PM, Szczesny B, Banerjee D, et al. Role of human DNA glycosylase Nei-like 2 (NEIL2) and single Strand break repair protein polynucleotide kinase 3 '-phosphatase in maintenance of mitochondrial genome. J Biol Chem. 2012;287:2819-29.

72. Shokolenko I, Venediktova N, Bochkareva A, Wilson GL, Alexeyev MF. Oxidative stress induces degradation of mitochondrial DNA. Nucleic Acids Res. 2009;37:2539-48.

73. Alexeyev M, Shokolenko I, Wilson G, LeDoux S. The maintenance of mitochondrial DNA integrity--critical analysis and update. Cold Spring Harb Perspect Biol. 2013;5:a012641

74. Vernon HJ, Bindoff LA. Mitochondrial ataxias. Handb Clin Neurol. 2018;155:129-41.

75. Wirtz S, Schuelke M. Region-specific expression of mitochondrial complex genes during murine brain development. PLoS One. 2011;6:e18897 Feany $M B$, editor.

76. Chakrabarti L, Zahra R, Jackson SM, Kazemi-Esfarjani P, Sopher BL, Mason $A G$, et al. Mitochondrial dysfunction in NnaD mutant flies and Purkinje cell degeneration mice reveals a role for Nna proteins in neuronal bioenergetics. Neuron. 2010;66:835-47.

77. Girard M, Lariviere R, Parfitt DA, Deane EC, Gaudet R, Nossova N, et al. Mitochondrial dysfunction and Purkinje cell loss in autosomal recessive spastic ataxia of Charlevoix-Saguenay (ARSACS). Proc Natl Acad Sci. 2012;109:1661-6.

78. Gao Y, Katyal S, Lee Y, Zhao J, Rehg JE, Russell HR, et al. DNA ligase III is critical for mtDNA integrity but not Xrcc1-mediated nuclear DNA repair. Nature. 2011;471:240-4.

79. Murai J, Huang SN, Das BB, Dexheimer TS, Takeda S, Pommier Y. TyrosylDNA phosphodiesterase 1 (TDP1) repairs DNA damage induced by topoisomerases I and II and base alkylation in vertebrate cells. J Biol Chem. 2012;287:12848-57.

80. Meagher M, Lightowlers RN. The role of TDP1 and APTX in mitochondrial DNA repair. Biochimie. 2014;100:121-4.

81. Fam HK, Choi K, Fougner L, Lim CJ, Boerkoel CF. Reactive oxygen species stress increases accumulation of tyrosyl-DNA phsosphodiesterase 1 within mitochondria. Sci Rep. 2018;8 Available from: http://www.nature.com/ articles/s41598-018-22547-8. [cited 2019 Mar 6].

82. Das BB, Dexheimer TS, Maddali K, Pommier Y. Role of tyrosyl-DNA phosphodiesterase (TDP1) in mitochondria. Proc Natl Acad Sci. 2010;107: 19790-5.

83. Katyal S, El-Khamisy SF, Russell HR, Li Y, Ju L, Caldecott KW, et al. TDP1 facilitates chromosomal single-strand break repair in neurons and is neuroprotective in vivo. EMBO J. 2007;26:4720-31.

84. Chiang S-C, Meagher M, Kassouf N, Hafezparast M, McKinnon PJ, Haywood $R$, et al. Mitochondrial protein-linked DNA breaks perturb mitochondrial gene transcription and trigger free radical-induced DNA damage. Sci Adv. 2017;3:e1602506

85. Ferro A, Carbone E, Zhang J, Marzouk E, Villegas M, Siegel A, et al. Shortterm succinic acid treatment mitigates cerebellar mitochondrial OXPHOS dysfunction, neurodegeneration and ataxia in a Purkinje-specific spinocerebellar ataxia type 1 (SCA1) mouse model. PLoS One. 2017;12: e0188425 Chakrabarti L, editor.

86. Ripolone M, Lucchini V, Ronchi D, Fagiolari G, Bordoni A, Fortunato F, et al. Purkinje cell COX deficiency and mtDNA depletion in an animal model of spinocerebellar ataxia type 1. J Neurosci Res. 2018;96:1576-85.

87. Laço MN, Oliveira CR, Paulson HL, Rego AC. Compromised mitochondrial complex II in models of Machado-Joseph disease. Biochim Biophys Acta (BBA) - Mol Basis Dis. 2012;1822:139-49.

88. Chou A-H, Yeh T-H, Kuo Y-L, Kao Y-C, Jou M-J, Hsu C-Y, et al. Polyglutamineexpanded ataxin-3 activates mitochondrial apoptotic pathway by upregulating Bax and downregulating Bcl-xL. Neurobiol Dis. 2006;21:333-45.

89. Goti D. A mutant Ataxin-3 putative-cleavage fragment in brains of Machado-Joseph disease patients and transgenic mice is cytotoxic above a critical concentration. J Neurosci. 2004;24:10266-79.

90. Hsu J-Y, Jhang Y-L, Cheng P-H, Chang Y-F, Mao S-H, Yang H-I, et al. The truncated C-terminal fragment of mutant ATXN3 disrupts mitochondria dynamics in spinocerebellar Ataxia type 3 models. Front Mol Neurosci. 2017; 10 Available from: http://journal.frontiersin.org/article/10.3389/fnmol.2017. 00196/full. [cited 2019 Jan 19].

91. Tzoulis C, Tran GT, Coxhead J, Bertelsen B, Lilleng PK, Balafkan N, et al. Molecular pathogenesis of polymerase gamma-related neurodegeneration: POLG-related neurodegeneration. Ann Neurol. 2014;76:66-81.
92. Abeti R, Parkinson MH, Hargreaves IP, Angelova PR, Sandi C, Pook MA, et al. Mitochondrial energy imbalance and lipid peroxidation cause cell death in Friedreich's ataxia. Cell Death Dis. 2016;7:e2237.

93. Chiang S, Kovacevic Z, Sahni S, Lane DJR, Merlot AM, Kalinowski DS, et al. Frataxin and the molecular mechanism of mitochondrial iron-loading in Friedreich's ataxia. Clin Sci. 2016;130:853-70.

94. Choi M, Kipps T, Kurzrock R. ATM mutations in Cancer: therapeutic implications. Mol Cancer Ther. 2016;15:1781-91.

95. Chow H-M, Cheng A, Song X, Swerdel MR, Hart RP, Herrup K. ATM is activated by ATP depletion and modulates mitochondrial function through NRF1. J Cell Biol. 2019;218:909-28.

96. Zhang Y, Lee J-H, Paull TT, Gehrke S, D'Alessandro A, Dou Q, et al. Mitochondrial redox sensing by the kinase ATM maintains cellular antioxidant capacity. Sci Signal. 2018;11:eaaq0702.

97. Valentin-Vega YA, Maclean KH, Tait-Mulder J, Milasta S, Steeves M, Dorsey FC, et al. Mitochondrial dysfunction in ataxia-telangiectasia. Blood. 2012;119: 1490-500.

98. Fang EF, Scheibye-Knudsen M, Brace LE, Kassahun H, SenGupta T, Nilsen H, et al. Defective Mitophagy in XPA via PARP-1 Hyperactivation and NAD +/SIRT1 reduction. Cell. 2014;157:882-96.

99. Manandhar M, Lowery MG, Boulware KS, Lin KH, Lu Y, Wood RD. Transcriptional consequences of XPA disruption in human cell lines. DNA Repair. 2017:57:76-90.

100. Scheibye-Knudsen M, Fang EF, Croteau DL, Bohr VA. Contribution of defective mitophagy to the neurodegeneration in DNA repair-deficient disorders. Autophagy. 2014;10:1468-9.

101. Hoch NC, Hanzlikova H, Rulten SL, Tétreault M, Komulainen E, Ju L, et al. XRCC1 mutation is associated with PARP1 hyperactivation and cerebellar ataxia. Nature. 2017;541:87-91.

102. Fang EF, Scheibye-Knudsen M, Chua KF, Mattson MP, Croteau DL, Bohr VA Nuclear DNA damage signalling to mitochondria in ageing. Nat Rev Mol Cell Biol. 2016;17:308-21.

103. Shull ERP, Lee $Y$, Nakane $H$, Stracker TH, Zhao J, Russell HR, et al. Differential DNA damage signaling accounts for distinct neural apoptotic responses in ATLD and NBS. Genes Dev. 2009;23:171-80.

104. Lee Y, Katyal S, Li Y, El-Khamisy SF, Russell HR, Caldecott KW, et al. The genesis of cerebellar interneurons and the prevention of neural DNA damage require XRCC1. Nat Neurosci. 2009;12:973-80.
Ready to submit your research? Choose BMC and benefit from:
- fast, convenient online submission
- thorough peer review by experienced researchers in your field
- rapid publication on acceptance
- support for research data, including large and complex data types
- gold Open Access which fosters wider collaboration and increased citations
- maximum visibility for your research: over $100 \mathrm{M}$ website views per year
At BMC, research is always in progress.
Learn more biomedcentral.com/submissions 